\title{
Quadrupole transitions near interface: general theory and application to atom inside a planar cavity
}

\author{
V.V.Klimov \\ P.N.Lebedev Physical Institute, Russian Academy of Sciences, \\ 53 Leninskii Prospect, 119991 Moscow , Russid* \\ M.Ducloy \\ Laboratoire de Physique des Lasers, \\ UMR CNRS 7538 Institut Galilee, Universite Paris-Nord, \\ Avenue J-B. Clement, F 93430 Villetaneuse, France
}

\begin{abstract}
Quadrupole radiation of an atom in an arbitrary environment is investigated within classical as well as quantum electrodynamical approaches. Analytical expressions for decay rates are obtained in terms of Green's function of Maxwell equations. The equivalence of both approaches is shown. General expressions are applied to analyze the quadrupole decay rate of an atom placed between two half spaces with arbitrary dielectric constant. It is shown that in the case when the atom is close to the surface, the total decay rate is inversely proportional to the fifth power of distance between an atom and a plane interface.
\end{abstract}

*Electronic address: vklim@sci.lebedev.ru 


\section{INTRODUCTION}

In the recent years the goal of science now has been moving from consideration of the fundamental properties of atoms to controlling and changing these properties. It is well known that decay rates of atoms can be changed in cavities 1]. Many investigations, have been devoted to a description of the cavity QED effects [2]. The main attention was paid there to the allowed (dipole) transitions. The decay rates of dipole transitions were investigated in the vicinity of spherical, cylinder, cone, spheroid, aperture, and more complicated nanobodies [3], [4]].

However, the influence of environment on the forbidden (quadrupole) transitions is also of great interest. First of all, it can help one to study the forbidden transitions. Second, with the help of the forbidden transitions one can describe the long-living states, which are, in turn, very important in many applications (quantum computers, quantum information). Finally, the atoms or molecules with forbidden (quadrupole) transitions can be used as detectors of field inhomogeneites.

The first experiment dealing with quadrupole transitions near a plane interface was carried out not along ago. The influence of interface on the absorption of Cs $6^{2} S_{1 / 2}-5^{2} D_{5 / 2}$ transition was studied [5],[6], [7]. As for the theoretical works, there were very few analyses

on this topic. In [8], [9], the classical calculations of decay rates of quadrupole transitions near the plane dielectric interface were performed. The quadrupole transitions near sphere and cylinder were considered within both the classical and QED approaches, and it was shown that both approaches gave identical results, as shown in [10],11]. However, there was no exact proof of equivalence between the classical and QED pictures.

The aim of this paper is to find expressions for the quadrupole decay rates and to prove their equivalence in an arbitrary environment. In Section [I] we derive expressions for total decay rate of a Lorenz oscillator in arbitrary environment through Green function of Maxwell equations. In Section 【II we find the expressions for total decay rate of an atom in arbitrary environment within the QED approach, and show that they are the same as those in the classical approach. Then we apply general results to find expressions for quadrupole decay rates for an atom placed in a planar cavity (Section IV]) This problem is very important for some experiments on reflection spectroscopy in thin cells [12]. General expressions for quadrupole decay rates in planar cavity are investigated for the case of dielectric or metallic 
walls in Section $\mathrm{V}$

\section{CLASSICAL DESCRIPTION OF QUADRUPOLE RADIATION IN ANY EN- VIRONMENT}

It is easy to show within classical electrodynamics that the total rate of work performed by the field can be presented in the form of [13]

$$
\frac{d E}{d t}=\int d^{3} \mathbf{r J}(\mathbf{r}, t) \cdot \mathbf{E}(\mathbf{r}, t)
$$

where $J(\mathbf{r}, t)$ and $E(\mathbf{r}, t)$ are the density of current and strength of the electric field, respectively. This power represents a conversion of electromagnetic field into mechanical or

thermal energy. In quasi monochromatic case we have instead of (11) the following expression

$$
\frac{d E}{d t}=\frac{1}{2} R e \int d^{3} \mathbf{r} \mathbf{J}^{*}(\mathbf{r}, \omega) \cdot \mathbf{E}(\mathbf{r}, \omega)
$$

where $\omega$ is the frequency, and $*$ means the complex conjugation.

The electric field can be expressed through current with the help of retarded Green function:

$$
E_{i}(\mathbf{r} ; \omega)=\frac{i}{\omega} \int d^{3} \mathbf{r}^{\prime} G_{i j}^{R}\left(\mathbf{r}, \mathbf{r}^{\prime} ; \omega\right) J_{j}\left(\mathbf{r}^{\prime} ; \omega\right)
$$

Here and below the lower Latin subscripts denote Cartesian coordinates and are to be summed over when repeated.

The retarded Green function (3) is the solution of Helmholtz wave equation

$$
\nabla \times\left(\nabla \times \overleftrightarrow{G}^{R}\left(\mathbf{r}, \mathbf{r}^{\prime} ; \omega\right)\right)-\left(\frac{\omega}{c}\right)^{2} \varepsilon(\mathbf{r}) \overleftrightarrow{G^{R}}\left(\mathbf{r}, \mathbf{r}^{\prime} ; \omega\right)=4 \pi\left(\frac{\omega}{c}\right)^{2} \overleftrightarrow{1} \delta \mathbf{r}-\mathbf{r}^{\prime}
$$

where $\varepsilon(\mathbf{r})$ stands for dielectric constant of environment, and for simplicity we assume that the media are nonmagnetic and nondispersive.

Substituting (33) in (21) the expression for power can be presented in the form

$$
\frac{d E}{d t}=-\frac{1}{2 \omega} \operatorname{Im} \int d^{3} \mathbf{r} \int d^{3} \mathbf{r}^{\prime} J_{i}^{*}(\mathbf{r}, \omega) G_{i j}^{R}\left(\mathbf{r}, \mathbf{r}^{\prime} ; \omega\right) J_{j}\left(\mathbf{r}^{\prime} ; \omega\right)
$$


To compare classical and quantum calculations it is convenient to consider stored energy as $E_{0}=\hbar \omega$. As a result the expression for decay rate will take the following form

$$
\gamma_{\text {tot }}^{\text {class }}=\frac{1}{E_{0}} \frac{d E}{d t}=-\frac{1}{2 \hbar \omega^{2}} \operatorname{Im} \int d^{3} \mathbf{r} \int d^{3} \mathbf{r}^{\prime} J_{i}^{*}(\mathbf{r}, \omega) G_{i j}^{R}\left(\mathbf{r}, \mathbf{r}^{\prime} ; \omega\right) J_{j}\left(\mathbf{r}^{\prime} ; \omega\right)
$$

For the relative decay rate we will have respectively

$$
\frac{\gamma_{\text {tot }}^{\text {class }}}{\gamma_{\text {tot }, 0}^{\text {class }}}=\frac{\operatorname{Im} \int d^{3} \mathbf{r} \int d^{3} \mathbf{r}^{\prime} J_{i}^{*}(\mathbf{r}, \omega) G_{i j}^{R}\left(\mathbf{r}, \mathbf{r}^{\prime} ; \omega\right) J_{j}\left(\mathbf{r}^{\prime} ; \omega\right)}{\operatorname{Im} \int d^{3} \mathbf{r} \int d^{3} \mathbf{r}^{\prime} J_{i}^{*}(\mathbf{r}, \omega) G_{i j}^{R, 0}\left(\mathbf{r}, \mathbf{r}^{\prime} ; \omega\right) J_{j}\left(\mathbf{r}^{\prime} ; \omega\right)}
$$

where $\gamma_{\text {tot }, 0}^{\text {class }}$ and $G_{i j}^{R, 0}\left(\mathbf{r}, \mathbf{r}^{\prime} ; \omega\right)$ are the total decay rate and the Green function in uniform (free) space, respectively,

$$
G_{i j}^{R, 0}\left(\mathbf{r}, \mathbf{r}^{\prime}, \omega\right)=\left[k^{2}\left(\delta_{i j}-\mathbf{n}_{i} \mathbf{n}_{j}\right) \frac{1}{\left|\mathbf{r}-\mathbf{r}^{\prime}\right|}+\left(3 \mathbf{n}_{i} \mathbf{n}_{j}-\delta_{i j}\right)\left(\frac{1}{\left|\mathbf{r}-\mathbf{r}^{\prime}\right|^{3}}-\frac{i k}{\left|\mathbf{r}-\mathbf{r}^{\prime}\right|^{2}}\right)\right] e^{i k\left|\mathbf{r}-\mathbf{r}^{\prime}\right|}
$$

In (18), $\mathbf{n}=\frac{\mathbf{r}-\mathbf{r}^{\prime}}{\left|\mathbf{r}-\mathbf{r}^{\prime}\right|}$ is the unit vector in the direction from the atom to the observation point and $k=\omega / c$ is the wave vector of free space.

In the case of quadrupoles the current and charge densities have the following form

$$
\begin{gathered}
\rho^{Q}=\frac{1}{3 !} \sum_{i, j} Q_{i j} \nabla_{i} \nabla_{j} \delta\left(\mathbf{r}-\mathbf{r}^{\prime}\right) \\
j_{i}^{Q}=\frac{i \omega}{3 !} \sum_{i, j} Q_{i j} \nabla_{j} \delta\left(\mathbf{r}-\mathbf{r}^{\prime}\right)
\end{gathered}
$$

where $\boldsymbol{r}$ is the radius-vector of the observation point, $\boldsymbol{r}^{\prime}$ is the radius-vector of the quadrupole position, and $Q_{i j}$ is the traceless quadrupole momentum tensor

$$
Q_{i j}=\int d \mathbf{x} \rho(\mathbf{x})\left(3 x_{i} x_{j}-x^{2} \delta_{i j}\right) .
$$

As is known [13], any quadrupole can be built out of two dipoles that are equal in amplitude and opposite in orientation. In Fig 1 some quadrupoles and the related quadrupole momenta are shown.

Now, after a substitution of (10) into (6) and (7) and a partial integration, the final expressions for full and relative decay rates will take the following form

$$
\gamma_{\text {tot }}^{\text {class } Q}=\frac{1}{72 \hbar} \operatorname{Im} \lim _{\mathbf{r} \rightarrow \mathbf{r}^{\prime}} Q_{i j}^{*} Q_{k l} \nabla_{j} \nabla_{l}^{\prime} G_{i k}^{R}\left(\mathbf{r}, \mathbf{r}^{\prime} ; \omega\right)
$$




$$
\frac{\gamma_{\text {tot }}^{\text {clas }, Q}}{\gamma_{\text {tot }, 0}^{\text {class } Q}}=\frac{\operatorname{Im} \lim _{\mathbf{r} \rightarrow \mathbf{r}^{\prime}} Q_{i j}^{*} Q_{k l} \nabla_{j} \nabla_{l}^{\prime} G_{i k}^{R}\left(\mathbf{r}, \mathbf{r}^{\prime} ; \omega\right)}{\operatorname{Im} \lim _{\mathbf{r} \rightarrow \mathbf{r}^{\prime}} Q_{i j}^{*} Q_{k l} \nabla_{j} \nabla_{l}^{\prime} G_{i k}^{R, 0}\left(\mathbf{r}, \mathbf{r}^{\prime} ; \omega\right)}
$$

In (12), (13) and hereafter, $\nabla, \nabla^{\prime}$ mean the differentiation over $\mathbf{r}$ or $\mathbf{r} /$, respectively. By calculating the limit in (12) for free-space Green function (8), we obtain that the expression for quadrupole decay rate in free space gets the following simple form

$$
\gamma_{\text {tot }, 0}^{\text {class } Q}=\frac{k^{5}}{360 \hbar} \sum_{i j}\left|Q_{i j}\right|^{2}
$$

Substituting this expression into (13) we obtain the following expression for relative quadrupole decay rate

$$
\frac{\gamma_{\text {tot }}^{\text {class } Q}}{\gamma_{\text {tot }, 0}^{\text {class }, Q}}=5 \frac{\operatorname{Im} \lim _{\mathbf{r} \rightarrow \mathbf{r}^{\prime}} Q_{i j}^{*} Q_{k l} \nabla_{j} \nabla_{l}^{\prime} G_{i k}^{R}\left(\mathbf{r}, \mathbf{r}^{\prime} ; \omega\right)}{k^{5} \sum_{i j}\left|Q_{i j}\right|^{2}}
$$

Thus, to calculate quadrupole decay rates it is suffice to determine the respective derivatives of Green function of Helmholtz wave equation. This result was quite expected as we know that electric quadrupoles interact with field gradients.

\section{QED DESCRIPTION OF QUADRUPOLE RADIATION IN ANY ENVIRON- MENT: LINEAR RESPONSE THEORY}

To calculate the quadrupole decay rate in arbitrary environment we use the work of ref.[14], 15], but apply a minimal coupling Hamiltonian with generalized Coulomb gauge

$$
\begin{aligned}
& H_{i n t}=-\frac{e}{2 m c}(\hat{\mathbf{p}} \hat{\mathbf{A}}(\mathbf{r})+\hat{\mathbf{A}} \hat{\mathbf{p}}(\mathbf{r}))+\frac{e^{2}}{2 m c^{2}} \hat{\mathbf{A}}^{2} \\
& \operatorname{div}(\varepsilon(\mathbf{r}) \hat{\mathbf{A}}(\mathbf{r}))=0, \quad \varphi=0
\end{aligned}
$$

Here $\hat{\mathbf{p}}$ is the operator of electron linear momentum and $\hat{\mathbf{A}}(\mathbf{r})$ is the vector potential at the electron position $\mathbf{r}$. The last term in (16) gives no contribution to calculation of decay rates.

Assuming that the matrix element of the electron momentum between initial $|i\rangle$ and final 
$|f\rangle$ states is zero, that is, $\langle f|\hat{\mathbf{p}}| i\rangle=0$, the Hamiltonian (16) can be presented in the form

$$
H_{i n t}=-\frac{e}{2 m c} \frac{\partial}{\partial r_{0, j}} A_{i}\left(\mathbf{r}^{\prime}\right)\left(\hat{p}_{i}\left(r_{j}-r_{j}^{\prime}\right)+\left(r_{j}-r_{j}^{\prime}\right) \hat{p}_{i}\right)
$$

where $\mathbf{r}^{\prime}$ is the vector of atom position.

In first order, the transition rate from initial atomic state $|i\rangle$ to a final state $|f\rangle$ is given by Fermi's golden rule [16],

$$
\begin{aligned}
& R_{f i}=\frac{2 \pi}{\hbar}\left(\frac{e}{m c}\right)^{2} \lim _{\mathbf{r} \rightarrow \mathbf{r}^{\prime}} \frac{\partial}{\partial r_{j}} \frac{\partial}{\partial r_{j^{\prime}}^{\prime}} D_{j i}^{f i} D_{j^{\prime} i^{\prime}}^{i f} \sum_{I, F} p(I)\left\langle I\left|A_{i}(\mathbf{r})\right| F\right\rangle\left\langle F\left|A_{i^{\prime}}\left(\mathbf{r}^{\prime}\right)\right| I\right\rangle \times \\
& \delta\left(E_{F}+E_{f}-E_{I}-E_{i}\right)
\end{aligned}
$$

where $D_{j i}^{f i}=\left\langle f\left|\left(\left(r_{j}-r^{\prime}{ }_{j}\right) \hat{p}_{i}\right)\right| i\right\rangle$ and capital letters denote eigenstates of the rest of the total system under consideration, neglecting its interaction with the atom of interest. Such eigenstates might involve, and depend on the coupling between the radiation field, other atoms, surface excitations, and the like. For convenience, we refer to those as the "field states". For simplicity we assume here that the field is in thermal equilibrium at a temperature $T$; $p(I)=\exp \left(-\beta E_{I}\right) / \sum_{k} \exp \left(-\beta E_{k}\right)$ with $\beta=\left(k_{B} T\right)^{-1}$, is the probability that the field is in state I.

Expressing the $\delta$ function of (18) in the integral form we find

$$
R_{f i}=\frac{1}{\hbar^{2}}\left(\frac{e}{m c}\right)^{2} \lim _{\mathbf{r} \rightarrow \mathbf{r}^{\prime}} \frac{\partial}{\partial r_{j}} \frac{\partial}{\partial r_{j^{\prime}}^{\prime}} \int_{-\infty}^{\infty} d t\left\langle A_{i}(\mathbf{r}, t) A_{i^{\prime}}\left(\mathbf{r}^{\prime}, 0\right)\right\rangle D_{j i}^{f i} D_{j^{\prime} i^{\prime}}^{i f} \exp \left(i \omega_{0} t\right)
$$

where $\omega_{0}=\left(E_{f}-E_{i}\right) / \hbar$. In Eq. (19) angular brackets indicate an ensemble average and $\mathrm{A}\left(\mathbf{r}_{0}, t\right)$ is an interaction picture operator, evolving as if (16) were not present,

$$
\mathbf{A}(\mathbf{r}, t)=\exp \left(\frac{i}{\hbar} H_{0} t\right) \mathbf{A}(\mathbf{r}, t) \exp \left(-\frac{i}{\hbar} H_{0} t\right)
$$

In (20) $H_{0}$ is the Hamiltonian of the whole system without interaction.

Further, one can rewrite (19) as Fourier component of the two-point correlation function, $G_{i i^{\prime}}^{A}\left(\mathbf{r}, \mathbf{r}^{\prime} ; t\right)=\left\langle A_{i}(\mathbf{r}, t) A_{i^{\prime}}\left(\mathbf{r}^{\prime}, 0\right)\right\rangle$

$$
R_{f i}=\frac{1}{\hbar^{2}}\left(\frac{e}{m c}\right)^{2} \lim _{\mathbf{r} \rightarrow \mathbf{r}^{\prime}} \frac{\partial}{\partial r_{j}} \frac{\partial}{\partial r_{j^{\prime}}^{\prime}} G_{i i^{\prime}}^{A}\left(\mathbf{r}, \mathbf{r}^{\prime} ; \omega_{0}\right) D_{j i}^{f i} D_{j^{\prime} i^{\prime}}^{i f}
$$


As in our gauge $\mathbf{E}=-\frac{1}{c} \dot{\mathbf{A}}$ it is possible to show, that (21) can be presented as

$$
R_{f i}=\frac{1}{\hbar^{2}}\left(\frac{e}{m \omega_{0}}\right)^{2} \lim _{\mathbf{r} \rightarrow \mathbf{r}^{\prime}} \frac{\partial}{\partial r_{j}} \frac{\partial}{\partial r_{j^{\prime}}^{\prime}} G_{i i^{\prime}}^{E}\left(\mathbf{r}, \mathbf{r}^{\prime} ; \omega_{0}\right) D_{j i}^{f i} D_{j^{\prime} i^{\prime}}^{i f}
$$

where $G_{i i^{\prime}}^{E}\left(\mathbf{r}, \mathbf{r}^{\prime} ; t\right)=\left\langle E_{i}(\mathbf{r}, t) E_{i^{\prime}}\left(\mathbf{r}^{\prime}, 0\right)\right\rangle$ is the two-point correlation function of electric field. It is convenient to express it through the retarded Green function defined as

$$
G_{i i^{\prime}}^{R}\left(\mathbf{r}, \mathbf{r}^{\prime} ; t\right)=\frac{i}{\hbar}\left\langle\left[E_{i}(\mathbf{r}, t) E_{i^{\prime}}\left(\mathbf{r}^{\prime}, 0\right)\right]\right\rangle \Theta(t)
$$

In (23) square brackets mean a commutator and $\Theta(t)$ is the Heaviside step function.

By applying the fluctuation-dissipation theorem [17] we obtain

$$
R_{f i}=\frac{1}{\hbar}\left(\frac{e}{m \omega_{0}}\right) \lim _{\mathbf{r} \rightarrow \mathbf{r}^{\prime}} \frac{\partial}{\partial r_{j}} \frac{\partial}{\partial r_{j^{\prime}}^{\prime}} D_{j i}^{f i} D_{j^{\prime} i^{\prime}}^{i f} \frac{2 I m G_{i i^{\prime}}^{R}\left(\mathbf{r}, \mathbf{r}^{\prime} ; \omega_{0}\right)}{\left[1-\exp \left(-\beta \hbar \omega_{0}\right]\right.}
$$

where

$$
G_{i i^{\prime}}^{R}\left(\mathbf{r}, \mathbf{r}^{\prime} ; \omega_{0}\right)=\int_{-\infty}^{\infty} d t G_{i i^{\prime}}^{R}\left(\mathbf{r}, \mathbf{r}^{\prime} ; t\right) \exp \left(i \omega_{0} t\right)
$$

is the Fourier component of retarded Green function of electric field.

The temperature dependence, which appears in the form of an occupation number will be important only for $\left(k_{B} T\right) \geq \hbar \omega_{0}$. Since we are interested primarily in the atomic transition energies of the order of a Rydberg, we can set $T=0 \mathrm{~K}$ in this equation. As a result the quadrupole decay rate will have the following form for $T=0$ :

$$
R_{f i}=\frac{2}{\hbar}\left(\frac{e}{m \omega_{0}}\right) \lim _{\mathbf{r} \rightarrow \mathbf{r}^{\prime}} \frac{\partial}{\partial r_{j}} \frac{\partial}{\partial r_{j^{\prime}}^{\prime}} D_{j i}^{f i} D_{j^{\prime} i^{\prime}}^{i f} \operatorname{Im} G_{i i^{\prime}}^{R}\left(\mathbf{r}, \mathbf{r}^{\prime} ; \omega_{0}\right)
$$

As $G_{i i^{\prime}}^{R}\left(\mathbf{r}, \mathbf{r}^{\prime} ; \omega\right)$ describes the response of the system, it is possible to show that this function is the solution of Maxwell equations [18]:

$$
\nabla \times\left(\nabla \times \overleftrightarrow{G^{k}}\left(\mathbf{r}, \mathbf{r}^{\prime} ; \omega\right)\right)-\left(\frac{\omega}{c}\right)^{2} \varepsilon(\mathbf{r}) \overleftrightarrow{G^{R}}\left(\mathbf{r}, \mathbf{r}^{\prime} ; \omega\right)=4 \pi\left(\frac{\omega}{c}\right)^{2} \overleftrightarrow{1} \delta\left(\mathbf{r}-\mathbf{r}^{\prime}\right)
$$

For quadrupole transitions with changing of principal or orbital quantum number the following identity is true

$$
\left\langle f\left|x_{i} \frac{\partial}{\partial x_{j}}\right| i\right\rangle=\frac{m \omega_{f i}}{2 \hbar}\left\langle f\left|x_{i} x_{j}\right| i\right\rangle
$$


Substituting it into eq (24) and using definition of quadrupole momentum $Q_{i j}^{f i}=$ $e\left\langle\left(3 x_{i} x_{j}-x^{2} \delta_{i j}\right)\right\rangle_{f i}$ we obtain the following expression for decay rates for arbitrary quadrupole transitions

$$
R_{f i}^{Q}=\frac{1}{18 \hbar} \lim _{r \rightarrow r^{\prime}} \nabla_{j} \nabla_{l}^{\prime} Q_{j i}^{f i} Q_{l k}^{i f} \operatorname{Im} G_{i k}^{R}\left(\mathbf{r}, \mathbf{r}^{\prime} ; \omega_{0}\right)
$$

where $G_{j k}^{R}\left(\mathbf{r}, \mathbf{r}^{\prime} ; \omega_{0}\right)$ is the retarded Green function of Maxwell equation (27).

It is very important to remember that this expression is valid for any media, including media with losses.

The quadrupole decay rate in free space is described by the same expression but with free space of the Green function $G_{j k}^{R, 0}\left(\mathbf{r}, \mathbf{r}^{\prime} ; \omega_{0}\right)$, instead

$$
R_{f i}^{Q, 0}=\frac{1}{18 \hbar} \lim _{r \rightarrow r^{\prime}} \nabla_{j} \nabla_{l}^{\prime} Q_{j i}^{f i} Q_{l k}^{i f} \operatorname{Im} G_{i k}^{R, 0}\left(\mathbf{r}, \mathbf{r}^{\prime} ; \omega_{0}\right)=\frac{k^{5}}{90 \hbar} \sum_{i j}\left|Q_{i j}\right|^{2}
$$

As a result relative decay rates gets the following form

$$
\frac{R_{f i}^{Q}}{R_{f i}^{Q, 0}}=5 \frac{\lim _{r \rightarrow r^{\prime}} \nabla_{j} \nabla_{l}^{\prime} Q_{j i}^{f i} Q_{l k}^{i f} \operatorname{Im} G_{i k}^{R}\left(\mathbf{r}, \mathbf{r}^{\prime} ; \omega_{0}\right)}{k^{5} \sum_{i j}\left|Q_{i j}\right|^{2}}
$$

Comparing expression (31) with the classical expression (15) one can see that they are identical. It means that both classical and QED models are equivalent for description of the total decay rate. Comparison of (301) and (14) reveals the difference by the factor of 4.The same difference takes place in the case of dipole transitions and is related to different definitions of dipole and quadrupole momenta in classical and quantum mechanics.

One should also remember that these equations describe the total decay rates. To find the radiative decay rates one should use other approaches, which allow one to take into account the radiation patterns of photons. It can be done, for example, within the classical approach.

\section{QUADRUPOLE DECAY RATES OF AN ATOM PLACED IN A PLANAR CAVITY}

To calculate the decay rates of quadrupole transition in an atom placed between two dielectric half-spaces (Fig. 2 ) one should find the electric Green function of Maxwell equa- 
tion. It is very important to ensure that this function should satisfy the symmetry condition and the Lorenz reciprocity relation, which follows from the definition (23). The approach suggested in [19] allows one to build such a function. According to [19] the Green function in layered media can be presented in the following form $\left(z>z^{\prime}\right)$

$$
\begin{aligned}
& G\left(\mathbf{r}, \mathbf{r}^{\prime} ; \omega\right)=\int \frac{d^{2} \mathbf{k}}{(2 \pi)^{2}} e^{i \mathbf{k}\left(\rho-\rho^{\prime}\right)} G\left(\mathbf{k}, z, z^{\prime} ; \omega\right) \\
& G\left(\mathbf{k}, z, z^{\prime} ; \omega\right)=\frac{2 \pi i}{\beta_{1}} \frac{k_{1}^{2}}{\varepsilon_{1}} e^{i \beta_{1} L} \sum_{q=p, s} \xi_{q} \frac{E_{q 1}^{>}(\mathbf{k}, \omega, z) E_{q 1}^{<}\left(-\mathbf{k}, \omega, z^{\prime}\right)}{1-r_{12}^{q} r_{13}^{q} e^{2 i \beta_{1} L}}
\end{aligned}
$$

In (32), $E_{q 1}^{>}(\mathbf{k}, \omega, z), E_{q 1}^{<}\left(-\mathbf{k}, \omega, z^{\prime}\right)$ are the mode functions

$$
\begin{aligned}
& E_{q 1}^{>}(\mathbf{k}, \omega, z)=\hat{\mathbf{e}}_{q 1}^{+}(\mathbf{k}) e^{i \beta_{1}(z-L)}+r_{12}^{q} \hat{\mathbf{e}}_{q 1}^{-}(\mathbf{k}) e^{-i \beta_{1}(z-L)} \\
& E_{q 1}^{<}\left(\mathbf{k}, \omega, z^{\prime}\right)=\hat{\mathbf{e}}_{q 1}^{-}(\mathbf{k}) e^{-i \beta_{1} z}+r_{13}^{q} \hat{\mathbf{e}}_{q 1}^{+}(\mathbf{k}) e^{i \beta_{1} z}
\end{aligned}
$$

and

$$
\begin{aligned}
& \hat{\mathbf{e}}_{p 1}^{ \pm}(\mathbf{k})=\frac{1}{k_{1}}\left(\mp \beta_{1} \hat{\mathbf{k}}+k \hat{\mathbf{z}}\right)=\hat{\mathbf{e}}_{p 1}^{\mp}(-\mathbf{k}) \\
& \hat{\mathbf{e}}_{s 1}^{ \pm}(\mathbf{k})=\hat{\mathbf{k}} \times \hat{\mathbf{z}}=-\hat{\mathbf{e}}_{s 1}^{\mp}(-\mathbf{k})
\end{aligned}
$$

Here $\beta_{j}=\sqrt{k_{j}^{2}-k^{2}}=\sqrt{\varepsilon_{j} k_{0}^{2}-k^{2}}\left(k_{0}=\omega / c\right)$ is the longitudinal wave vector and $r_{12}^{q}, r_{13}^{q}$ are the conventional Fresnel reflection coefficients

$$
r_{i j}^{p}=\frac{\varepsilon_{j} \beta_{i}-\varepsilon_{i} \beta_{j}}{\varepsilon_{j} \beta_{i}+\varepsilon_{i} \beta_{j}}, r_{i j}^{s}=\frac{\beta_{i}-\beta_{j}}{\beta_{i}+\beta_{j}}
$$

for $\mathrm{p}$ and s polarized waves, and $L$ is the distance between plane interfaces.

Now by substituting this function into (29) and integrating it over angle $\varphi$ in $\mathrm{x}-\mathrm{y}$ plane $\left(\hat{k}_{x}=\cos \varphi, \hat{k}_{y}=\sin \varphi\right)$ we obtain the expression of the quadrupole decay rate.

In the case of the z-oriented quadrupole, that is in the case when

$$
\mathbf{Q}=Q_{z z}\left[\begin{array}{ccc}
-1 / 2 & 0 & 0 \\
0 & -1 / 2 & 0 \\
0 & 0 & 1
\end{array}\right]
$$

the decay rate in free space according to (30) gets the following form

$$
\gamma_{z z}^{0}=\frac{k_{0}^{5} Q_{z z}^{2}}{60}
$$


and the expression for relative decay rate has the following form

$$
\left(\frac{\gamma}{\gamma_{0}}\right)_{z z}^{Q}=\frac{15}{2 k_{0}^{5}} \operatorname{Re} \int_{0}^{\infty} k^{3} d k \beta_{1} \frac{\left(1-r_{12}^{p} e^{2 i \beta_{1} s}\right)\left(1-r_{13}^{p} e^{2 i \beta_{1} z_{0}}\right)}{\left(1-r_{12}^{p} r_{13}^{p} e^{2 i \beta_{1} L}\right)}
$$
[8]

In the case of a single interface with $\left(\left(L-z_{0}\right)=s \rightarrow \infty\right)$ we have a more simple result

$$
\left(\frac{\gamma}{\gamma_{0}}\right)_{z z}^{Q}=\frac{15}{2 k_{0}^{5}} \operatorname{Re} \int_{0}^{\infty} k^{3} d k \beta_{1}\left(1-r_{13}^{p} e^{2 i \beta_{1} z_{0}}\right)=1-\frac{15}{2 k_{0}^{5}} \operatorname{Re} \int_{0}^{\infty} k^{3} d k \beta_{1} r_{13}^{p} e^{2 i \beta_{1} z_{0}}
$$

This coincidence is very interesting because the Green function used for calculation of decay rate[8] is asymmetric.

In the case of $x y+y x$ quadrupole or in the case of $x x-y y$ quadrupole, where

$$
\begin{gathered}
\mathbf{Q}=Q_{x y}\left[\begin{array}{lll}
0 & 1 & 0 \\
1 & 0 & 0 \\
0 & 0 & 0
\end{array}\right] \\
\mathbf{Q}=Q_{x x}\left[\begin{array}{ccc}
1 & 0 & 0 \\
0 & -1 & 0 \\
0 & 0 & 0
\end{array}\right]
\end{gathered}
$$

the decay rates in free space according to (30) get the following form

$$
\begin{aligned}
& \gamma_{x y}^{0}=\frac{k_{0}^{5} Q_{x y}^{2}}{45} \\
& \gamma_{x x}^{0}=\frac{k_{0}^{5} Q_{x x}^{2}}{45}
\end{aligned}
$$

and for the relative decay rate we have, respectively,

$$
\left(\frac{\gamma}{\gamma_{0}}\right)_{x y}^{Q}=\left(\frac{\gamma}{\gamma_{0}}\right)_{x x}^{Q}=\frac{5}{4 k_{0}^{5}} \operatorname{Re} \int_{0}^{\infty} \frac{k^{3} d k}{\beta_{1}}\left[\begin{array}{c}
\beta_{1}^{2} \frac{\left(1-r_{12}^{p} e^{2 i \beta_{1} s}\right)\left(1-r_{13}^{p} e^{2 i \beta_{1} z_{0}}\right)}{\left(1-r_{12}^{p} r_{13}^{p} e^{2 i \beta_{1} L}\right)} \\
+k_{1}^{2} \frac{\left(1+r_{12}^{s} e^{2 i \beta_{1} s}\right)\left(1+r_{13}^{s} e^{2 i \beta_{1} z_{0}}\right)}{\left(1-r_{12}^{s} r_{13}^{s} e^{2 i \beta_{1} L}\right)}
\end{array}\right]
$$

In the case of $s \rightarrow \infty$, that is in the case of single interface, we have a more simple result 
8]

$$
\begin{aligned}
& \left(\frac{\gamma}{\gamma_{0}}\right)_{z z}^{Q}=\frac{5}{4 k_{0}^{5}} \operatorname{Re} \int_{0}^{\infty} \frac{k^{3} d k}{\beta_{1}}\left[\beta_{1}^{2}\left(1-r_{13}^{p} e^{2 i \beta_{1} z_{0}}\right)+k_{1}^{2}\left(1+r_{13}^{s} e^{2 i \beta_{1} z_{0}}\right)\right] \\
& =1+\frac{5}{4 k_{0}^{5}} \operatorname{Re} \int_{0}^{\infty} \frac{k^{3} d k}{\beta_{1}}\left[k_{1}^{2} r_{13}^{s}-\beta_{1}^{2} r_{13}^{p}\right] e^{2 i \beta_{1} z_{0}}
\end{aligned}
$$

Finally, in the case of $x z+z x$ or $y z+z y$ quadrupoles, where

$$
\begin{aligned}
& \mathbf{Q}=Q_{x z}\left[\begin{array}{lll}
0 & 0 & 1 \\
0 & 0 & 0 \\
1 & 0 & 0
\end{array}\right] \\
& \mathbf{Q}=Q_{y z}\left[\begin{array}{lll}
0 & 0 & 0 \\
0 & 0 & 1 \\
0 & 1 & 0
\end{array}\right]
\end{aligned}
$$

the decay rates in free space according to (30) get the following form

$$
\begin{aligned}
& \gamma_{x z}^{0}=\frac{k_{0}^{5} Q_{x z}^{2}}{45} \\
& \gamma_{y z}^{0}=\frac{k_{0}^{5} Q_{y z}^{2}}{45}
\end{aligned}
$$

and for relative decay rate we respectively have

$$
\left(\frac{\gamma}{\gamma_{0}}\right)_{x z}^{Q}=\left(\frac{\gamma}{\gamma_{0}}\right)_{y z}^{Q}=\frac{5}{4 k_{0}^{5}} \operatorname{Re} \int_{0}^{\infty} \frac{k d k}{\beta_{1}}\left[\begin{array}{c}
\left(\beta_{1}^{2}-k^{2}\right)^{2} \frac{\left(1+r_{12}^{p} e^{2 i \beta_{1} s}\right)\left(1+r_{13}^{p} e^{2 i \beta_{1} z_{0}}\right)}{\left(1-r_{12}^{p} r_{13}^{p} e^{2 i \beta_{1} L}\right)} \\
+\beta_{1}^{2} k_{1}^{2} \frac{\left(1-r_{12}^{s} e^{2 i \beta_{1} s}\right)\left(1-r_{13}^{s} e^{2 i \beta_{1} z_{0}}\right)}{\left(1-r_{12}^{s} r_{13}^{s} e^{2 i \beta_{1} L_{1}}\right)}
\end{array}\right]
$$
[8]

In the case $s \rightarrow \infty$, that is, in the case of single interface, we have a more simple result

$$
\begin{aligned}
& \left(\frac{\gamma}{\gamma_{0}}\right)_{x z}^{Q}=\left(\frac{\gamma}{\gamma_{0}}\right)_{y z}^{Q}=\frac{5}{4 k_{0}^{5}} \operatorname{Re} \int_{0}^{\infty} \frac{k d k}{\beta_{1}}\left[\left(\beta_{1}^{2}-k^{2}\right)^{2}\left(1+r_{13}^{p} e^{2 i \beta_{1} z_{0}}\right)+\beta_{1}^{2} k_{1}^{2}\left(1-r_{13}^{s} e^{2 i \beta_{1} z_{0}}\right)\right]= \\
& 1+\frac{5}{4 k_{0}^{5}} \operatorname{Re} \int_{0}^{\infty} \frac{k d k}{\beta_{1}}\left[\left(\beta_{1}^{2}-k^{2}\right)^{2} r_{13}^{p}-r_{13}^{s} \beta_{1}^{2} k_{1}^{2}\right] e^{2 i \beta_{1} z_{0}}
\end{aligned}
$$

As mentioned above, these results describe the total decay rates, i.e. radiative and nonradiative. Generally, it is difficult to separate these contributions. However, one can assume that this separation can be made on the basis of the classical energy flux method. It should be noted that a purely radiation channel may exist, in this geometry, in an ideal 
case of matter without losses. At negligibly small losses, the radiation energy would not go to infinity. This is the difference between the geometry under consideration and an open geometry, at which the radiation might go to infinity throughout a free space.

\section{ANALYSIS OF RESULTS AND ILLUSTRATIONS}

The expressions that had been obtained in the previous section are rather complicated and their calculation is an independent problem, in a general case. The complexity is due to the fact that the integrands are the complex functions with a set of the singular points, which might be both the branching points, and the poles. These peculiarities are connected with physical properties of the problem. In any case, in the integrand there are the branching points at $k= \pm \sqrt{\varepsilon} k_{0}$. If the mode wave propagation is formed in a cavity (metallic mirrors) then the poles appear in the integrands. So, in different physical situations, the calculations are to be performed with account of these factors.

\section{A. Atom between perfect metallic mirrors}

In the case of the well conducting metallic mirrors, the expressions (38), (43), and (48), in which the reflection coefficients are substituted by their analogs for the case of an ideal conductivity,

$$
r^{p}=1, r^{s}=-1
$$

will be good approximations for the rates

The expressions for the decay rates of quadrupole states may be reduced to the form

$$
\begin{gathered}
\left(\frac{\gamma}{\gamma_{0}}\right)_{z z}^{Q}=\frac{15}{k_{0}^{5}} \operatorname{Im} \int_{0}^{\infty} k^{3} d k \beta_{1} \frac{\sin \left(\beta_{1} s\right) \sin \left(\beta_{1} z_{0}\right)}{\sin \left(\beta_{1} L\right)} \\
\left(\frac{\gamma}{\gamma_{0}}\right)_{x y}^{Q}=\left(\frac{\gamma}{\gamma_{0}}\right)_{x x}^{Q}=\frac{5}{2 k_{0}^{5}} \operatorname{Im} \int_{0}^{\infty} \frac{k^{3} d k}{\beta_{1}}\left[\beta_{1}^{2}+k_{1}^{2}\right] \frac{\sin \left(\beta_{1} s\right) \sin \left(\beta_{1} z_{0}\right)}{\sin \left(\beta_{1} L\right)}
\end{gathered}
$$




$$
\left(\frac{\gamma}{\gamma_{0}}\right)_{x z}^{Q}=\left(\frac{\gamma}{\gamma_{0}}\right)_{y z}^{Q}=-\frac{5}{2 k_{0}^{5}} \operatorname{Im} \int_{0}^{\infty} \frac{k d k}{\beta_{1}}\left[\left(\beta_{1}^{2}-k^{2}\right)^{2}+\beta_{1}^{2} k_{1}^{2}\right] \frac{\cos \left(\beta_{1} s\right) \cos \left(\beta_{1} z_{0}\right)}{\sin \left(\beta_{1} L\right)}
$$

Because the ideal conductivity is the limiting case for a real metal, where the poles must lie above the horizontal axis of integration, the integration circuit of the ideal conductivity must envelope the poles from below, as shown in Fig 3 .

To calculate the integrals (51)-(53) it is convenient to use the variable $\beta_{1}=\sqrt{k_{0}^{2}-k^{2}}$,

$$
\begin{gathered}
\left(\frac{\gamma}{\gamma_{0}}\right)_{z z}^{Q}=\frac{15}{k_{0}^{5}} \operatorname{Im} \int_{i \infty}^{k_{0}}\left(k_{0}^{2}-\beta_{1}^{2}\right) d \beta_{1} \beta_{1}^{2} \frac{\sin \left(\beta_{1} s\right) \sin \left(\beta_{1} z_{0}\right)}{\sin \left(\beta_{1} L\right)} \\
\left(\frac{\gamma}{\gamma_{0}}\right)_{x y}^{Q}=\left(\frac{\gamma}{\gamma_{0}}\right)_{x x}^{Q}=\frac{5}{2 k_{0}^{5}} \operatorname{Im} \int_{i \infty}^{k_{0}}\left(k_{0}^{4}-\beta_{1}^{4}\right) d \beta_{1} \frac{\sin \left(\beta_{1} s\right) \sin \left(\beta_{1} z_{0}\right)}{\sin \left(\beta_{1} L\right)} \\
\left(\frac{\gamma}{\gamma_{0}}\right)_{x z}^{Q}=\left(\frac{\gamma}{\gamma_{0}}\right)_{y z}^{Q}=-\frac{5}{2 k_{0}^{5}} \operatorname{Im} \int_{i \infty}^{k_{0}} d \beta_{1}\left[\left(2 \beta_{1}^{2}-k_{0}^{2}\right)^{2}+\beta_{1}^{2} k_{0}^{2}\right] \frac{\cos \left(\beta_{1} s\right) \cos \left(\beta_{1} z_{0}\right)}{\sin \left(\beta_{1} L\right)}
\end{gathered}
$$

where the path of integration is shown in Fig 3 . By calculating the integrals (54)-(156) with the residue theorem one can obtain the following results

$$
\begin{gathered}
\left(\frac{\gamma}{\gamma_{0}}\right)_{z z}^{Q}=\frac{15 \pi}{\tilde{L}} \sum_{n=1}^{n_{\max }}\left(\frac{\pi n}{\tilde{L}}\right)^{2}\left(1-\left(\frac{\pi n}{\tilde{L}}\right)^{2}\right) \sin ^{2}\left(\frac{\pi n z_{0}}{L}\right) \\
\left(\frac{\gamma}{\gamma_{0}}\right)_{x y}^{Q}=\left(\frac{\gamma}{\gamma_{0}}\right)_{x x}^{Q}=\frac{5 \pi}{2 \tilde{L}} \sum_{n=1}^{n_{\max }}\left(1-\left(\frac{\pi n}{\tilde{L}}\right)^{4}\right) \sin ^{2}\left(\frac{\pi n z_{0}}{L}\right) \\
\left(\frac{\gamma}{\gamma_{0}}\right)_{x z}^{Q}=\left(\frac{\gamma}{\gamma_{0}}\right)_{y z}^{Q}=\frac{5 \pi}{2 \tilde{L}}\left[\frac{1}{2}+\sum_{n=1}^{n_{\max }}\left\{\left(1-2\left(\frac{\pi n}{\tilde{L}}\right)^{2}\right)^{2}+\left(\frac{\pi n}{\tilde{L}}\right)^{2}\right\} \cos ^{2}\left(\frac{\pi n z_{0}}{L}\right)\right]
\end{gathered}
$$

where $n_{\max }=[\widetilde{L} / \pi]$ is integral part of $\widetilde{L} / \pi$, and $\widetilde{L}=k_{0} L$

Figure 4 illustrates the quadrupole decay rates in a resonator formed by a hypothetic metal with $\varepsilon=-200+0.01 i$ in respect to the position and orientation of a quadrupole. As seen from the Figure, the asymptotic expressions (57)-(59) approximate well the exact expressions (38), (43) (48), excluding the region that is in a close proximity to the metal 
surface. However, in the vicinity of the surface, the nonradiative losses connected with imaginary part of the dielectric constant are of the main importance. These losses are the reason of a fast increase in the total losses (see Eqs.(64)-(66)). In the case of an ideal conductor, the losses are absent, and there is a difference between the decay rates of a hypothetic metal and an ideal conductor.

In the case of real metals, that difference might be still more profound because the imaginary part of the permittivity is not negligibly small as compared to the real part. Figure 5 illustrates the decay rates for a micro-resonator with silver mirrors. From the Figure one can see that the rate of spontaneous decays in the real resonator differs from the decay rate in the cavity with ideal walls substantially.

\section{B. Atom between dielectric mirrors}

A planar cavity can also be realized on the basis of two opposite dielectric half-spaces. No propagating waveguiding modes are formed in that case, and the integrand, respectively, has no poles in a complex plane near a real axis. This should simplify a numerical calculation of the integrals. Figures 67 demonstrate the dependencies of the quadrupole decay rates on the atomic position and structure of the quadrupole moment for a planar resonator with silica walls.

In the case of quite a large-size cavity (micro-cavity, Fig [6) one can observe an increase in the rate of spontaneous decays as an atom is approaching the wall. In contrast to the case of metallic mirrors, such an increase is due to the coupling of the non-propagating near fields emitted by the quadrupole, with the propagating fields inside the dielectric (silica). One can notice the influence of the intrinsic non-radiative processes at a distance less than $1 \mathrm{~nm}$ only, because the imaginary part of the quartz permittivity is very small at optical frequencies (see Eqs. (64)-(66) $)$. At such distances one should take into account the random inhomogeneities of the surface structure.

As the distance between dielectric walls is small (nano-cavity, Fig[7), the electric fields are near fields one, at any atom position between the walls, and there occurs the effective field transformation into the wave propagation over a dielectric. This provides a considerable acceleration of the transitions. The intrinsic non-radiative decay channel is formed at distances closer to the wall, and this is unseen on the picture. 
Note that all the energy of an excited atom will be emitted in the dielectric, and all the losses will, therefore, be non-radiative. But in the case of the weakly absorbing dielectrics, including silica, it is not unreasonable to distinguish between the regions of the effective transformation into the propagating waves and the regions of the intrinsic radiative losses.

\section{Atom inside ultra thin cell}

Very interesting spectroscopy experiments are carried out now with atoms inside an extra-

thin dielectric cell. Suffice it to say that the width of the cell cab be as small as $20 \mathrm{~nm}$ [20]. So, it is very interesting to understand the behavior of decay rate in that case.

All dimensional parameters are small in comparison with wavelength. As a result we can use the quasi-static approximation to calculate decay rates found in a previous section. The quasistatic approach here is equivalent to the case of $k>>k_{0}=\omega / c$. In this limit the Fresnel reflection coefficients can be simplified substantially

$$
\begin{aligned}
& r_{12}^{p}=r_{13}^{p}=r=\frac{\varepsilon-1}{\varepsilon+1} \\
& r_{12}^{s}=r_{13}^{s}=0
\end{aligned}
$$

As a result the decay rate in the small width of cavity case will have the following form:

$$
\begin{aligned}
& \left(\frac{\gamma}{\gamma_{0}}\right)_{z z}^{Q}=-\frac{45}{8} \operatorname{Im} \sum_{n=0}^{\infty} r^{2 n+1}\left(\frac{2 r}{[\tilde{L}(n+1)]^{5}}-\frac{1}{[\tilde{s}+\tilde{L} n]^{5}}-\frac{1}{\left[\tilde{z}_{0}+\tilde{L} n\right]^{5}}\right) \\
& \left(\frac{\gamma}{\gamma_{0}}\right)_{x y}^{Q}=-\frac{15}{16} \operatorname{Im} \sum_{n=0}^{\infty} r^{2 n+1}\left(\frac{2 r}{[\tilde{L}(n+1)]^{5}}-\frac{1}{[\tilde{s}+\tilde{L} n]^{5}}-\frac{1}{\left[\tilde{z}_{0}+\tilde{L} n\right]^{5}}\right) \\
& \left(\frac{\gamma}{\gamma_{0}}\right)_{x z}^{Q}=\frac{15}{16} \operatorname{Im} \sum_{n=0}^{\infty} r^{2 n+1}\left(\frac{2 r}{[\tilde{L}(n+1)]^{5}}+\frac{1}{[\tilde{s}+\tilde{L} n]^{5}}+\frac{1}{\left[\tilde{z}_{0}+\tilde{L} n\right]^{5}}\right)
\end{aligned}
$$

where $\tilde{z}_{0}, \tilde{s}, \tilde{L}$ stand for $k_{0} z_{0}, k_{0} s, k_{0} L$, respectively. In the case when atom is very close to one surface $\tilde{z}_{0}<<\tilde{L}$ only one term $(\mathrm{n}=0)$ is important in this series

$$
\left(\frac{\gamma}{\gamma_{0}}\right)_{z z}^{Q}=\frac{45}{8 \tilde{z}_{0}^{5}} I m r
$$




$$
\begin{aligned}
& \left(\frac{\gamma}{\gamma_{0}}\right)_{x y}^{Q}=\frac{15}{16 \tilde{z}_{0}^{5}} \mathrm{Imr} \\
& \left(\frac{\gamma}{\gamma_{0}}\right)_{x z}^{Q}=\frac{15}{16 \tilde{z}_{0}^{5}} \mathrm{Imr}
\end{aligned}
$$

From this asymptotics one can see that the total decay rate increases inversely proportional to the fifth power of distance to surface $z=0$. This behavior is different substantially from the dipole case, where decay rates increase inversely proportional to the third power of distance to surface. Another interesting point one can get from (64) - (66) , is that the $z z$-quadrupoles suffer a six-fold enhancement in comparison with other components.

\section{CONCLUSIONS}

In this article, the processes of the spontaneous quadrupole atomic radiation in an arbitrary environment were considered within the framework of both classical and quantum electrodynamics. The general equations derived for the rates of quadrupole transitions were expressed through the spatial derivatives of the retarded Green function corresponding to the classical problem of electrodynamics. It was shown that the expressions differ by a numerical coefficient 4 only, which is connected with the definition of quadrupole moments which have different physical sense in the classical and quantum mechanics. The expressions for the relative decay rates, i.e. the rates normalized by the uniform space rate, prove to be identical.

The results obtained are applied to a description of quadrupole atomic transitions in a planar cavity. The explicit analytical expressions for the rates of any quadrupole transition were found for such a cavity. The results have been analyzed in detail for the planar cavities with dielectric and metallic walls. It was found that the quadrupole transitions are accelerated with decreasing resonator size. In the case of dielectric walls, such an acceleration is due to the transformation of the near dipole fields into the propagating waves inside the dielectric. In the case of metallic mirrors, the acceleration becomes more profound, and is due to the radiation absorption at the surface layer of a metal.

In this paper we restrict ourselves to investigation of quadrupole decay rates. However, our approach can be also applied to description of frequency shifts of quadrupole transitions in nanoenviroment. Again, general expressions for frequency shift will be expressed 
through space derivatives of retarded Green function. We will present detailed investigation of frequency shifts of quadrupole transitions in nanoenviroment in a separate publication.

\section{Acknowledgments}

The authors thank the Russian Foundation for Basic Research, grant \# 04-02-16211 (V.K.), and Centre National de la Recherche Scientifique (V.K., M.D.) for their financial support of this work. One of the authors (V.K.) is grateful to the colleagues of the Laboratoire de Physique des Lasers (Universite Paris-Nord), where this work has been completed, for their hospitality. This work has been done as part of the European Union FASTNET consortium.

[1] E.M. Purcell, Spontaneous Emission Probabilities at Radio Frequencies, Phys. Rev. 69, 681 (1946).

[2] Cavity Quantum Electrodynamics, edited by P.Berman, (Academic, New York,1994).

[3] V.V. Klimov, M. Ducloy, and V.S. Letokhov, Spontaneous emission of an atom in the presence of nanobodies Kvantovaya Elektronika, 31, 569 (2001).

[4] V.V. Klimov, Spontaneous atomic radiation in presence of nanobodies, Physics Uspekhi, 46, $13(2003)$.

[5] Satoshi Tojo, Masahiro Hasuo, and Takashi Fujimoto, Absorption Enhancement of an Electric Quadrupole Transition of Cesium Atoms in an Evanescent Field, Phys.Rev.Lett., 92, 053001 (2004).

[6] Satoshi Tojo and Masahiro Hasuo, Oscillator-strength enhancement of electric-dipoleforbidden transitions in evanescent light at total reflection, Phys. Rev. A, 71, 012508 (2005).

[7] Satoshi Tojo, Takashi Fujimoto, and Masahiro Hasuo, Precision measurement of the oscillator strength of the cesium $6^{2} S_{1 / 2} \rightarrow 5^{2} D_{5 / 2}$ electric quadrupole transition in propagating and evanescent wave fields, Phys. Rev. A.,71, 012507 (2005).

[8] R.R.Chance, A. Prock, R.Silbey, Molecular fluorescence and energy transfer near interfaces, Adv. Chem. Phys., 37, 1 (1978). 
[9] R.R.Chance, A.Prock, R.Silbey, Comments on the classical theory of energy transfer. II Extension to higher multipoles and anisotropic media, J. Chem. Phys., 65, 2527 (1976).

[10] V.V.Klimov, M.Ducloy, Allowed and forbidden transitions in an atom placed near an ideally conducting cylinder, Phys. Rev. A, 62, 043818 (2000).

[11] V.V.Klimov, V.S.Letokhov. Quadrupole Radiation of an Atom Dipole in the vicinity of Dielectric Microsphere, Phys. Rev. A, 54, 440 (1996).

[12] D.Bloch, M.Ducloy, Atom-wall interaction, in Advances in Atomic.Molecular and Optical Physics ,50, 96(2005)

[13] J.A. Stratton, Electromagnetic Theory (New York: McGraw-Hill, 1941)

[14] J.M. Wylie and J.E.Sipe, Quantum electrodynamics near an interface, Phys. Rev. A, 30, 1185 (1984);

[15] J.M.Wylie and J.E.Sipe, Quantum electrodynamics near an interface, Phys. Rev. A, 32, 2030 (1985).

[16] E. Fermi,Quantum Theory of Radiation, Rev. Mod. Phys., 4, 87 (1932).

[17] A.S.Davydov, Quantum Mechanics, Moscow, Nauka, 1973 (In Russian)

[18] E.M.Lifshitz and L.P.Pitaevskii, Statistical Physics, (Moscow, Nauka) Part 2, p.374-377 (In Russian)

[19] M. S. Tomas, Green function for multilayers: Light scattering in planar cavities, Phys. Rev. A 51, 2545 (1995).

[20] G.Dutier et al," Coupling of atoms,surfaces and fields in dielectric nanocavities", in "Laser Spectroscopy XV" (World Scientific, Singapore, 2004) p.277.

[21] G. Hass and L. Hadley, Optical Properties of Metals. In: D.E. Gray, ed., American Institute of Physics Handbook, (New York, McGraw-Hill, 1963), p. 6. 


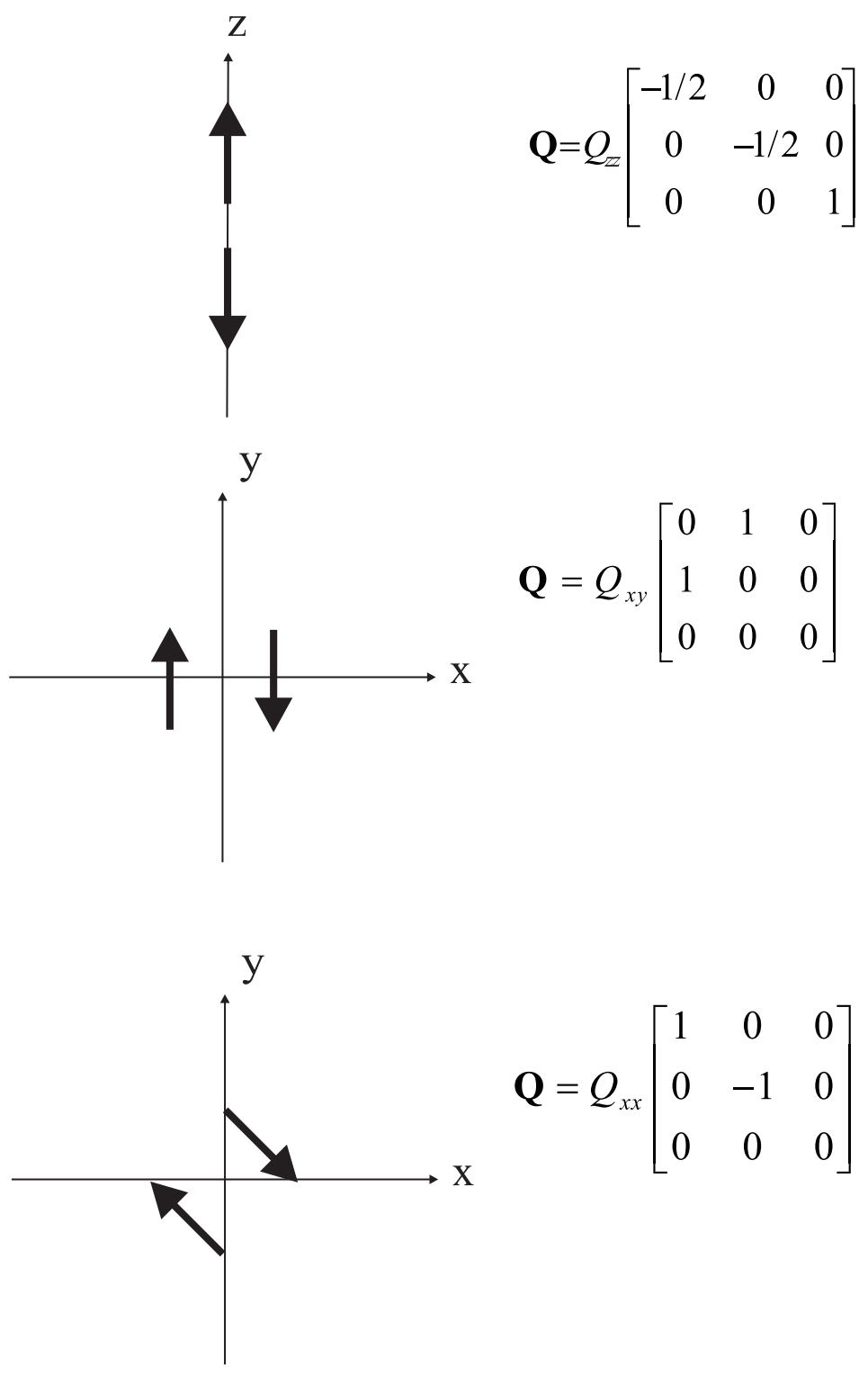

FIG. 1: Some kinds of quadrupoles and the corresponding quadrupole momentum tensors. 


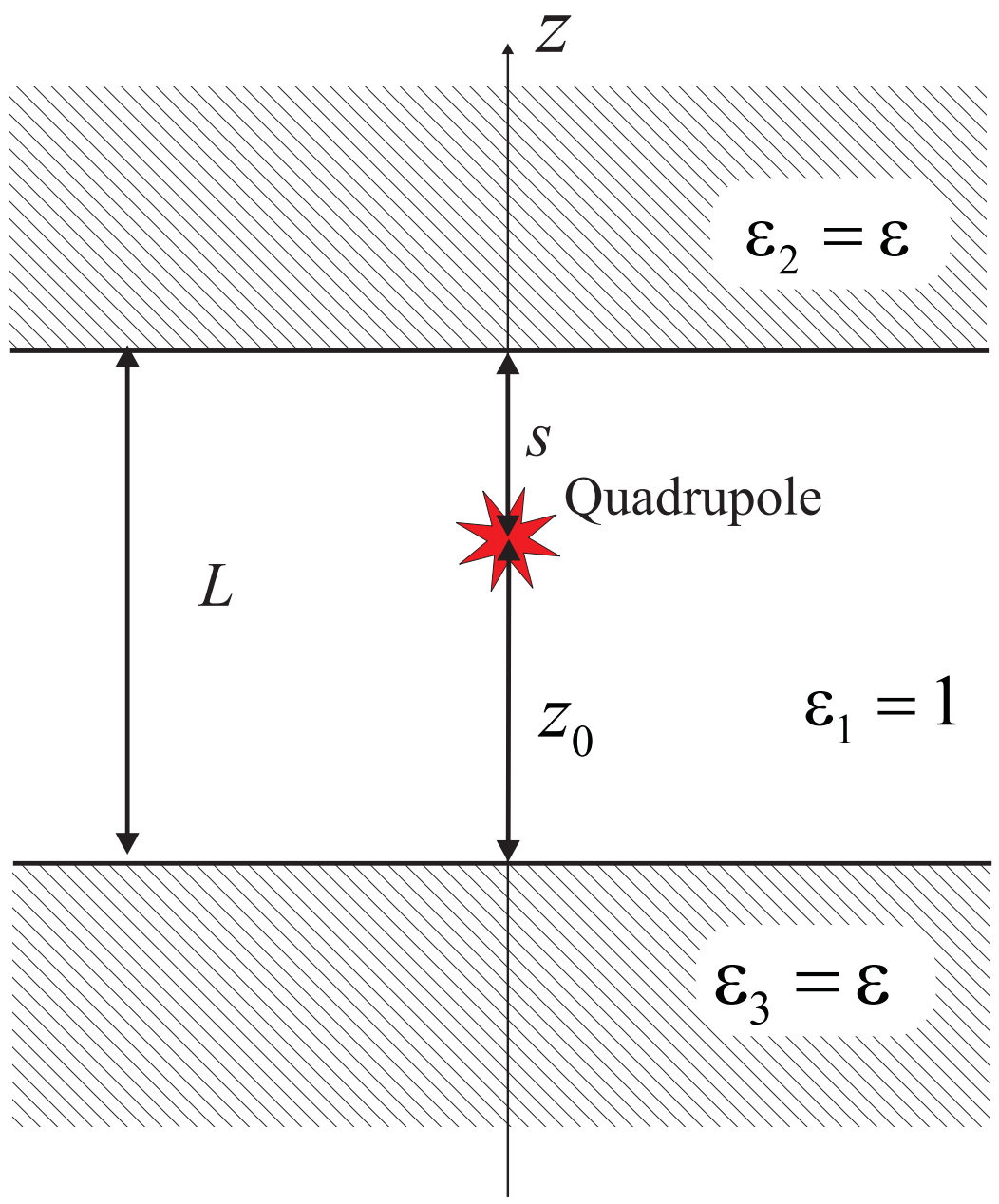

FIG. 2: Geometry of the problem of quadrupole radiation of an atom placed in a planar cavity. 

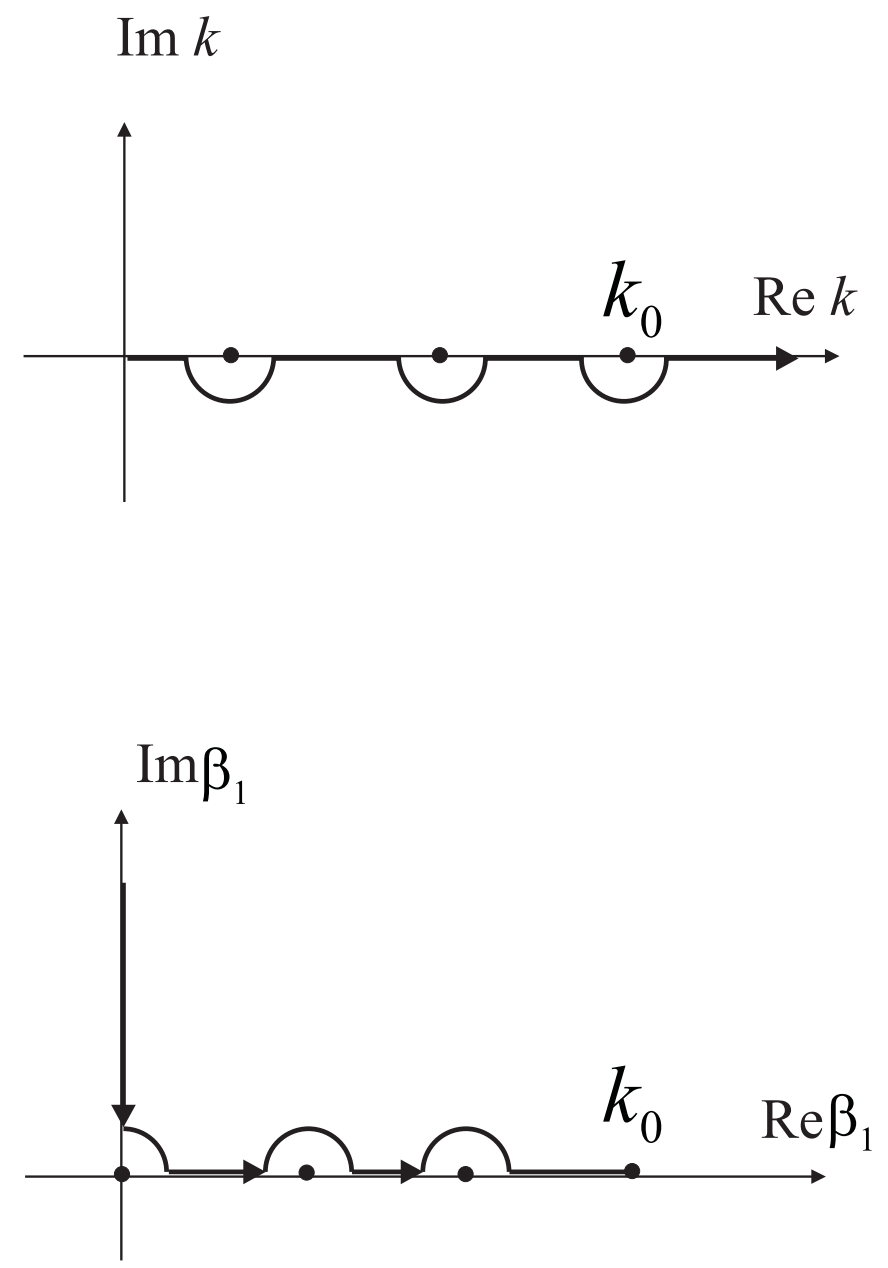

FIG. 3: Contours of integration in the case of ideally conducting mirrors. 


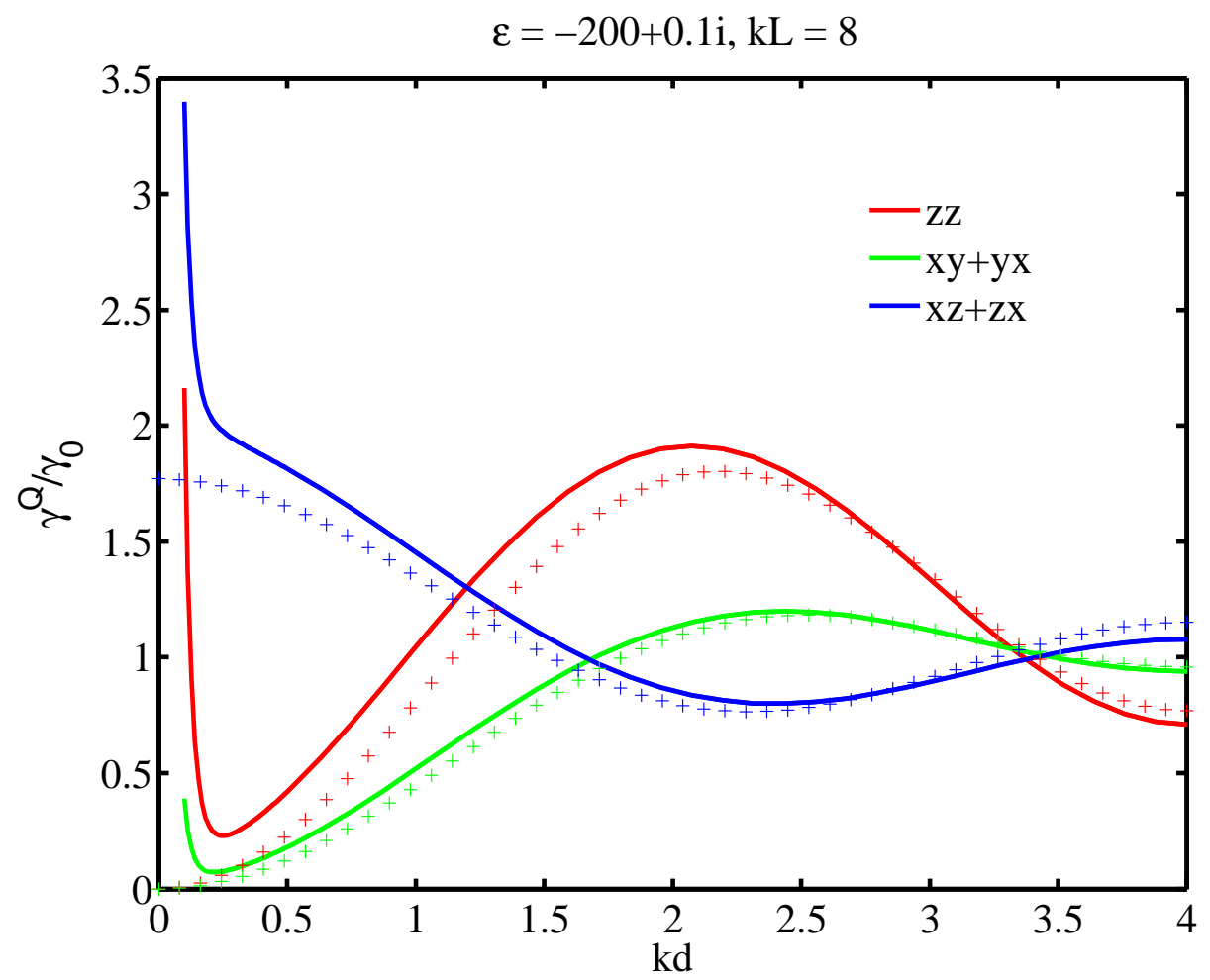

FIG. 4: The quadrupole decay rates of different quadrupoles versus their position in the case of a hypothetic material with $\epsilon=-200+0.1 i$, and in the case of an ideal conductivity (dotted line). 


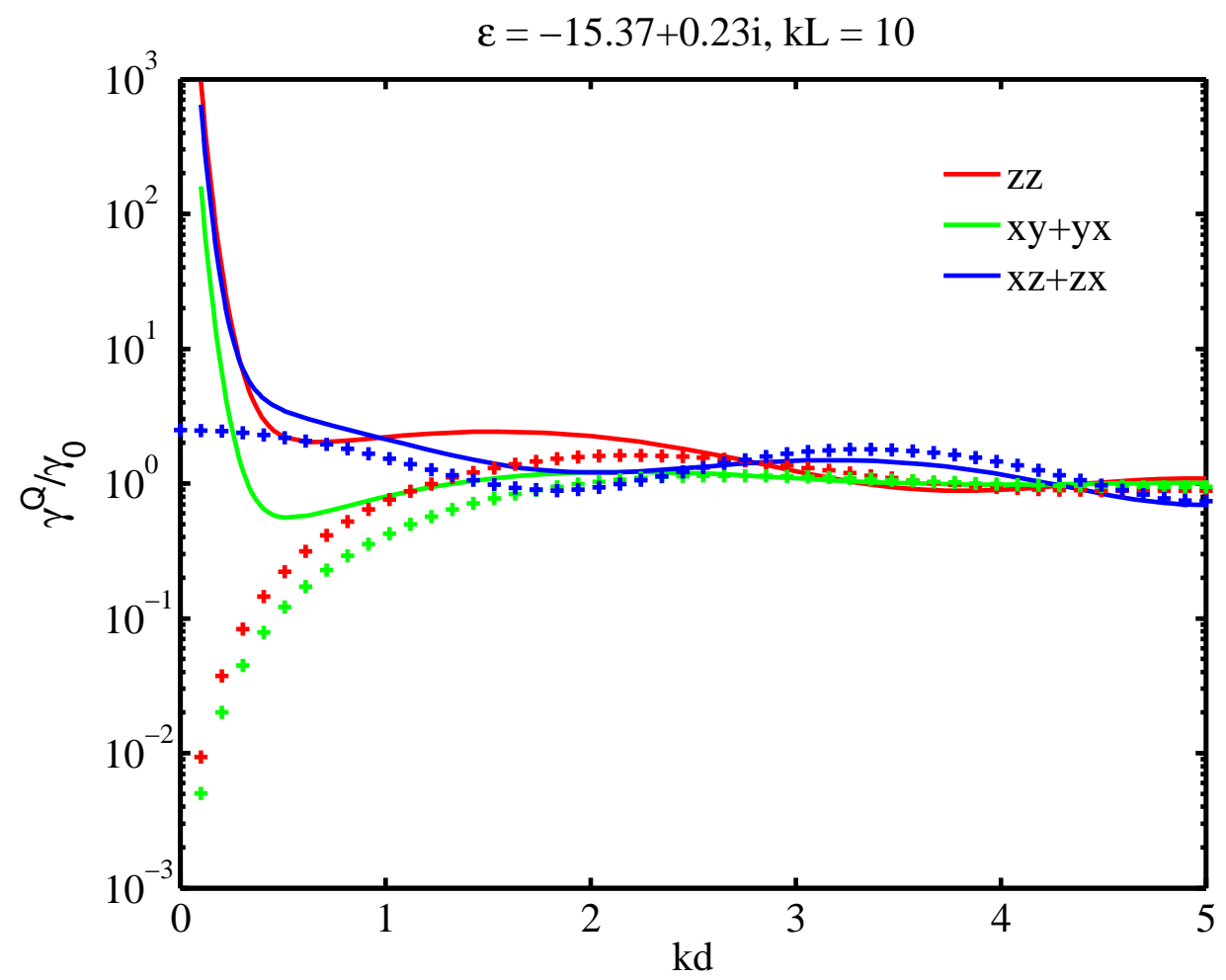

FIG. 5: The quadrupole decay rates of different quadrupoles versus their position between two thick silver $(A g: \epsilon=15.37+0.231 i, \lambda=632.8 \mathrm{~nm}$ [21]) mirrors (dotted lines correspond to the case of the ideally conducting walls). 


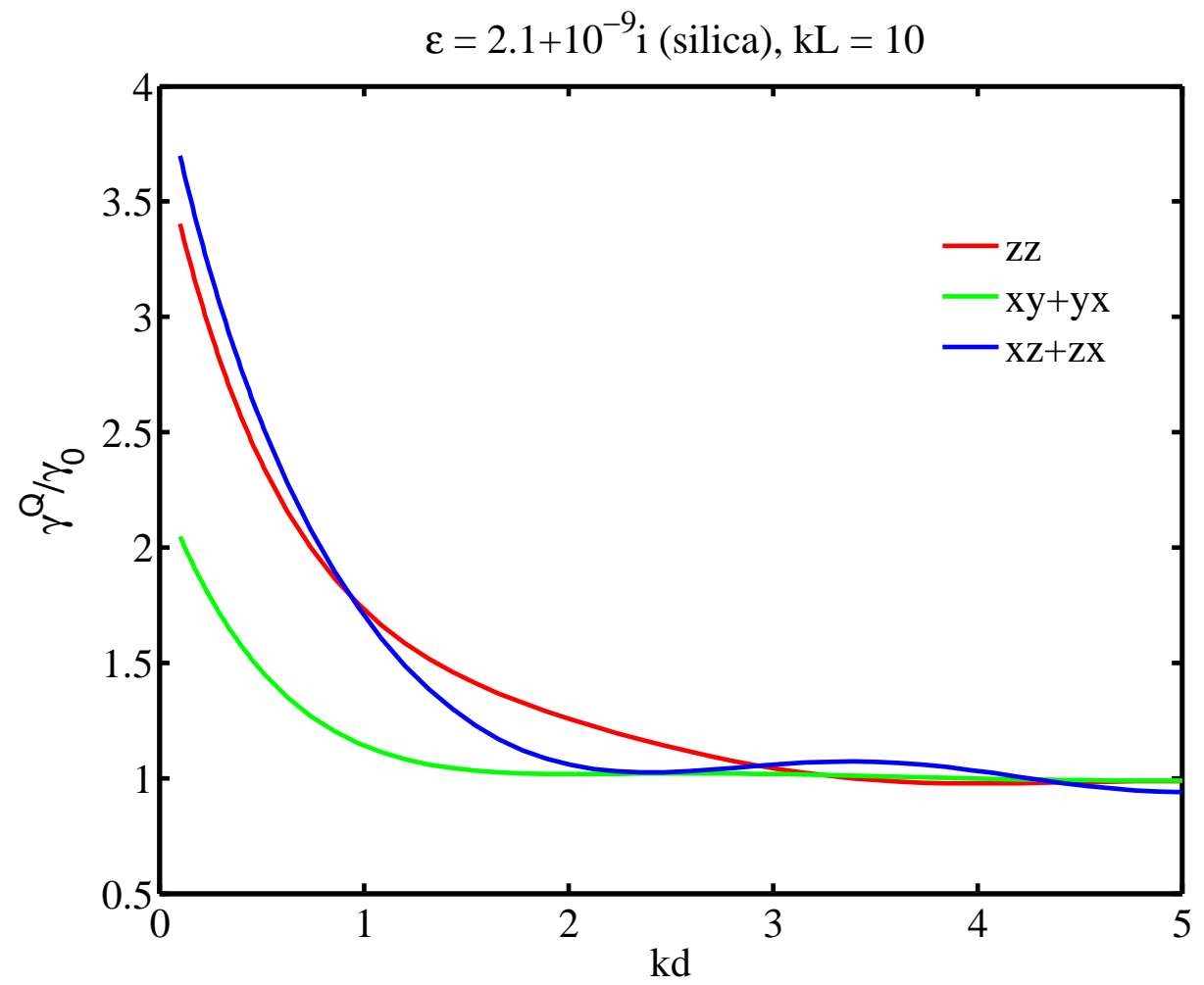

FIG. 6: The spontaneous decay rates of different quadrupoles versus their position between two quartz half-spaces with $\epsilon=2.1+0.000000001 i$ (silica) in the case of a micro-resonator $(k L=10)$. 


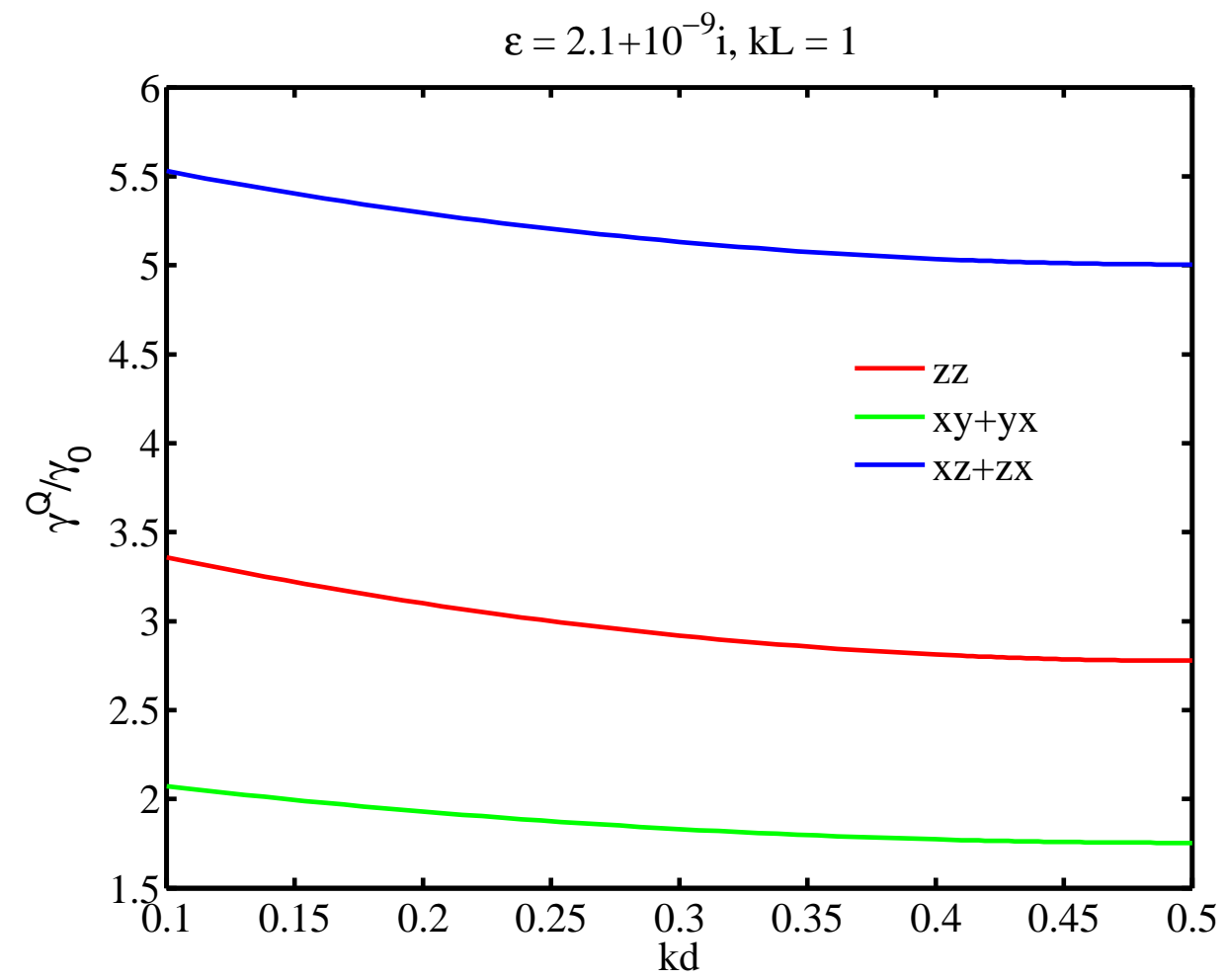

FIG. 7: The spontaneous decay rates of different quadrupoles versus their position between two quartz half-spaces with $\epsilon=2.1+0.000000001 i$ (silica) in the case of a nano-resonator $(k L=1)$. 